\title{
Vulvar lichen sclerosus: could a moisturizing and healing product reduce the prolonged use of topical steroid?
}

\author{
Filippo Murina*, Franco Vicariotto, Stefania Di Francesco and Silvia Oneda \\ Lower Genital Tract Disease Unit, V. Buzzi Hospital-University of Milan, Milan, Italy
}

\begin{abstract}
Background: Vulvar lichen sclerosus (LS) is an immune-mediated disease of unknown etiology causing itch, burning, and plaques with overlying excoriation and linear fissures. The standard treatment for this disease is the use of topical corticosteroids but evidence on the most appropriate treatment regimen is lacking.

Objective: To assess the effectiveness of a soft foam based on Tiab system (TF), complex of titanium micro crystals covalently bound with silver ions associated with hyaluronic acid, in the supportive treatment of lichen sclerosus .

Method: A total of 43 female patients with a clinical and/or histological diagnosis of LS were enrolled in the study. Of these women, 21 were treated with the topical application of mometasone furoate $0.1 \%$ (MMF) once daily for 4 weeks and then twice weekly for another 4 weeks in combination with a moisturizing cream , and 22 were treated with MMF once daily for 20 days and then only a soft foam TF once daily for the first 15 days and thereafter twice weekly for another 45 days . Clinical symptoms (itching and burning) and signs (vulvar suface disruption) were documented at baseline and at the end of the eight-week treatment.
\end{abstract}

Results: There was a significant reduction of symptoms between the basal scores and the symptoms complaints at 8-week treatment. Moreover, a more pronounced improvement in burning after 2 months was observed.

Conclusion: The present study shows that daily application of TF can be an effective adjunct for the treatment of LS. The foam had not only a moisturizer action, it may also be associated with a reduction in epithelial disruption in the form of excoriations and fissures.

\section{Introduction}

Lichen sclerosus (LS) is a chronic, progressive, inflammatory skin condition found most often in the anogenital region. The cause of disease is still under investigation. There is evidence to suggest that autoimmune factors may be involved in its pathogenesis and recent evidence has shown autoantibodies to extracellular matrix protein [1].

It was also found an increased frequency of other autoimmune disorders in females with LS [2], such as alopecia areata, vitiligo, thyroid disease, and pernicious anemia. Physical examination is important for differentiating LS from other causes of vulvar discomfort. The appearance of the vulva skin depends on the degree of disease progression. The skin may appear white, thickened, and excoriated, with edema and resorption of the labia minora, and stenosis of the introitus occurs as well as ulceration and fissuring [3].

The most common symptoms of vulvar dermatosis are pruritus, pain, dyspareunia, and postcoital soreness. The area involved may vary from a small, single area to the entire region of vulva, perineum, and perianus. There may be extension to the genitocrural folds, buttocks, and thighs. The characteristic sites involved are the interlabial sulci, labia minora and labia majora, clitoris and clitoral hood, and perineum and perianal area, giving rise to the characteristic 'figure-of-eight' shape. The diagnosis of LS is usually clinical. When the clinical features are typical, histologic examination is not always essential. Fragility is a hallmark of LS, becoming manifest in erosions, fissuring, purpura, and ecchymoses. Fissuring is especially common between the clitoris and urethra, in the interlabial sulci, and at the base of the posterior fourchette [4].
Tearing during sexual intercourse or physical examination is common. The clinical and histological alterations of vulvar dermatoses are responsible for changes in vulvar skin barrier function that enhance the symptoms (dehydration and impaired lipid skin film). Pain, soreness, and dysuria may be a consequence of erosions or fissures. A high proportion of women of all ages reported that LS led to significant sexual problems including dyspareunia and apareunia due to continuing inflammatory disease as well as due to anatomic changes and scarring from long-standing active disease [4]. The current accepted management guidelines for LS advocate the use of topical corticosteroids as first-line treatment with relief of symptoms and clinical and histologic improvement, and it is also recommended to use emollient creams with the aim of restoring the protective skin barrier $[5,6]$. For lack of sufficient long-term studies there are still concerns about the long-term safety of topical corticosteroids. Feared adverse effects of long-term, topical corticosteroid maintenance treatment are atrophy, rebound reactions, fungal infections, reactivation of human papilloma virus (HPV) and herpes simplex virus infection, or systemic absorption.

The aim to this study was to assess the effectiveness of a soft foam based on Tiab system (TF), complex of titanium micro crystals

Correspondence to: Filippo Murina M.D.; address: V. Buzzi Hospital, Via Castelvetro 32-Milano, Italy, Tel: +390257995464; E-mail: filippomurina@tin.it

Key words: vulvar dermatosis, lichen sclerosus, vulvar itching, moisturizer

Received: November 01, 2017; Accepted: November 15, 2017; Published: November 20, 2017 
covalently bound with silver ions associated with hyaluronic acid, in the supportive treatment of LS. Our previous study demonstrated that daily application of TF can be an effective adjunct for the treatment of vulvar dermatosis (LS and lichen simplex).

The foam not only had a moisturizer action, it may also be associated with a reduction in epithelial disruption in the form of excoriations and fissures. Our premise is that TF can acts not only as a moisturizing agent, but it may has a synergistic action with the topical steroid in promoting wound healing and restoration of the protective film skin altered in patients with LS, it aimed to reduce corticosteroid exposure time with a collateral improvement in patient compliance.

\section{Methods}

In this open-label, randomized controlled prospective study, women of 18 years or older with biopsy-proven LS who had the ability to sign written informed consent, willingness to comply with the study requirements, were recruited.

The following characteristics were recorded for all patients: historical features: age, ethnicity, menopausal status (premenopausal, postmenopausal without hormone therapy, or postmenopausal with hormone therapy), duration of symptoms, and previous treatment.

At $t=0$ subjective evaluations of itching, burning, and dyspareunia, the latter of which was evaluated only when applicable, were obtained by using a 0 to 3 score $(0=$ absent, $1=$ mild, $2=$ strong, $3=$ severe $)$. Clinical features of vulvar lesions were also collected. They were obtained by using a 0 to 3 score determined through the following characteristics: $0=$ Flat white lesions $(<30 \%$ of vulvar surface), $1=$ Flat and/or raised white/red lesions ( $>30<50 \%$ of vulvar surface), $2=$ Flat and/or raised white/red lesions ( $>30<50 \%$ of vulvar surface) plus erosions and/or fissures and

$3=$ Flat and/or raised white/red lesions ( $>50 \%$ of vulvar surface) plus erosions and/or fissures. Photographs of the LS lesions were used to evaluate and to measure their features.

Patients were randomized, using a computer-generated random number table, to either of two groups: one receiving topical mometasone furoate $0.1 \%$ (MMF) on the affected vulvar surfaces once daily for 4 weeks and then twice weekly for another 4 weeks in combination with a moisturizing cream; and one receiving topical mometasone furoate $0.1 \%$ (MMF) once daily for 20 days and then only a soft foam based on $\mathrm{Tiab}^{\circ}$ system (TF) once daily for the first 15 days and thereafter twice weekly for another 45 days. The signs and symptoms were classified before and after 60 days of treatment. The study was carried out in accordance with the applicable rules concerning the review of research ethics committees and informed consent. The EPI-INFO version 6.0 (Centers for Disease Control and Prevention, Atlanta, GA) was used for all statistical analyses. Descriptive statistical analyses (means, standard deviations, and percentages) were performed using the Student $t$ test. Differences were considered significant at $\mathrm{p} \leq .05$.

\section{Results}

A total of 43 female patients with a clinical and/or histological diagnosis of LS were enrolled in the study. Of these women, 21 were treated with the topical application of MMF + moisturizing cream and 22 were treated with the topical application of MMF $+\mathrm{TF}$.

The patients' demographic characteristics were comparable in the 2 groups in regards to age, disease duration before diagnosis, and symptoms score (Table 1). In terms of the subjective outcomes reported by the patients (itching, burning, and dyspareunia), there was a significant reduction in symptoms between the basal scores and the symptom complaints after 60 days of therapy (Table 2). However, we reported a more pronounced improvement in burning 2 months after treatment in the MMF+TF group compared to the MMF + moisturizing cream group $(\mathrm{p}=.04)$. The other symptom scores were not significantly different between the 2 treatment groups.

Moreover we observed a significant reduction of signs related to LS in both groups (Table 3). During the entire study no drop out related to the topical treatments was observed; only three patients experienced a mild transient burning after TF application.

\section{Discussion}

The goals of treatment for LS are to alleviate a woman's symptoms, prevent disease progression and scarring, provide long-term

Table 1. Personal and baseline characteristics of the subjects.

\begin{tabular}{|c|c|c|c|}
\hline Variable & MMF + moisturizing cream (n=21) & MMF + TF (n=22) & P \\
\hline Age (years) & $59.4(12.66)$ & $57.2(13.90)$ & $18.3(22.93)$ \\
\hline Duration of symptoms (months) & $18.4(22.22)$ & $1.92(0.98)$ & 0.98 \\
\hline Itching* $^{*}$ Burning* & $2.04(0.86)$ & $0.73(0.93)$ & 0.45 \\
\hline Dyspareunia* $^{*}$ & $0.76(0.85)$ & $0.24(0.48)$ \\
\hline
\end{tabular}

Data are presented as mean value \pm standard deviation. *Score according to a score from 0 to 3

$(0=$ absent; $1=$ mild; $2=$ strong; $3=$ severe $)$. Significance is taken at $\mathrm{p}<.05 . \mathrm{MMF}=$ mometasone furoate $0.1 \% ; \mathrm{TF}=$ foam based on Tiab ${ }^{\circledR}$ system $^{2}$

Table 2. Treatment outcome of itching, burning and dyspareunia after 60 days of therapy.

\begin{tabular}{|c|c|c|c|c|c|c|}
\hline & Itching* & $\mathrm{P}$ & Burning* & $\mathrm{P}$ & Dyspareunia* & $\mathrm{P}$ \\
\hline MMF + moisturizing cream $(n=21)$ & $0.36(0.76)$ & 0.001 & $0.49(0.80)$ & 0.001 & $0.32(066)$ & 0.001 \\
\hline MMF + TF $(n=22)$ & $0.10(0.37)$ & 0.001 & $0.24(0.48)$ & 0.001 & $0.27(0.49)$ & 0.001 \\
\hline
\end{tabular}

Data are presented as mean value \pm standard deviation. "Score according to a score from 0 to 3

$(0=$ absent $1=$ mild; $2=$ strong; $3=$ severe $)$. Significance is taken at $\mathrm{p}<.05 . \mathrm{MMF}=$ mometasone furoate $0.1 \% ; \mathrm{TF}=$ foam based on Tiab ${ }^{\circledR}$ system

Table 3. Treatment outcome of vulvar signs after 60 days of therapy.

\begin{tabular}{|c|c|c|c|c|}
\hline & Basal $^{*}$ & P & After 60 days $^{*}$ \\
\hline MMF + moisturizing cream (n=21) & $2.09(0.87)$ & 0.001 & $0.19(0.45)$ \\
\hline MMF + TF (n=22) & $2.15(0.91)$ & 0.001 & 0.001 \\
\hline
\end{tabular}

Data are presented as mean value \pm standard deviation. "See score in methods.

Significance is taken at $\mathrm{p}<.05 . \mathrm{MMF}=$ mometasone furoate $0.1 \% ; \mathrm{TF}=$ foam based on $\mathrm{Tiab}^{\circledR}$ system. 
surveillance, and prevent development vulvar cancer. LS can develop into widespread scarring and superficial ulcers. Scarring may result in labial fusion, narrowing of the vaginal opening, clitoral hood adhesion, and clitoral burying, thereby impairing or destroying sexual function. Urination may also become problematic and painful, and certain patients experience discomfort during defecation [7]. In addition to being a debilitating physical condition, LS can have detrimental consequences on affected women's psychosexual and psychosocial life.

Ultra-potent topical steroid medications are the mainstay of treatment for patients with LS and there is strong evidence supporting their efficacy. The most used is clobetasol propionate $0.05 \%$ ointment, but the less potent mometasone furoate has shown a similar efficacy [8]. We demonstrated that mometasone furoate has shown a reduction in mean symptom and sign scores compared with baseline in both treatments. It is of interest that patients in our study experienced a significant reduction in mean symptom scores after a relatively short administration schedule (20 days) compared to literature data, and maintenance therapy consisted of TF foam alone [3].

We can postulate that the soft foam based on Tiab system had not only a moisturizer action, it also accelerates normalization of the hydrolipidic film, a natural protective barrier for the skin that helps retain moisture and maintain the skin's natural suppleness, and it may also be associated with a reduction in epithelial disruption in the form of excoriations and fissures. In fact the foam formulation creates a protective barrier and it promotes normal scarring and healing through the creation of a favorable microenvironment to the cellular tissue repair [9]. In addition the foam based on Tiab system has a reservoir effect for lengthy maintenance of active ingredient in vulvar surface. Many of our patients achieved a considerable recovery of LSrelated signs.

A significant improvement of mean objective scores was obtained with both treatment regimens when compared with baseline. Consistently with these findings, MMF+TF regime also proved highly effective in treating the objective features of the disease. Most notably, in the present study, the TF treatment with a lower duration of time of MMF regimens did not differ significantly in any of the assessed efficacy end points with a long lasting MMF treatment.

For lack of sufficient long-term studies there are still concerns about the long-term safety of topical corticosteroids. Feared adverse effects of long-term, topical corticosteroid maintenance treatment are atrophy, striae formation, rebound reactions, fungal infections, reactivation of human papilloma virus (HPV) and herpes simplex virus infection, or systemic absorption.

During the trial no adverse events directly connected to the therapies were observed, thus the two group of treatment showed the same favourable safety profile and tolerability.
This is probably because of the implementation of a schedule that tends to reduce corticosteroid exposure time with a collateral improvement in patient compliance. Another strength of our study is the TF formulation. In fact, the new foam exists as a liquid pressurized in an aluminum can with a hydrocarbon propellant (propane/ butane), which upon valve actuation forms a foam lattice Patients find foams less dense and therefore generally easier to apply to and spread on the skin surface. This may have important clinical implications, especially if the diseased area is inflamed and overly sensitive to mechanical shearing forces. In addition, because the new foam leaves little or no residue after application, patients find the vehicle cosmetically attractive compared with the "greasy" sensation often experienced after the use of ointments.

This study has several limitations; first, the sample size is small; and secondly, the limited follow-up time. More work and longer observation is needed to verify whether TF treatment could prevent subsequent relapses and scar formation. Finally, the lack of universal parameters to assess disease severity and treatment response might influence the efficacy comparison of different treatments. In conclusion, corticosteroids represent the most appropriate agents for the reduction of symptom and sign of LS and the association of a new generation foam based on Tiab system enhance their efficacy and providing a reduction of topical steroids exposure.

\section{References}

1. Moyal-Barracco M, Wendling J (2014) Vulvar dermatosis. Best Prac Res Clin Obste Gynaecol 28: 946-958. http://dx.doi.org/10.1016/j.bpobgyn.2014.07.005

2. Perez-Lopez FR, Ceausu I, Depypere H, Erel CT, Lambrinoudaki I, et al. (2013) EMAS clinical guide: Vulvar lichen sclerosus in peri and postmenopausal women. Maturitas 74: 279-282. [Crossref]

3. Neill SM, Lewis FM, Tatnall FM, Cox NH; British Association of Dermatologists (2010) British Association of Dermatologists' Guidelines for the Management of Lichen Sclerosus 2010. Br J Dermatol 163: 672-682. [Crossref]

4. Kellogg Spadt S, Kusturiss E (2015) Vulvar dermatoses: A primer for the sexual medicine clinician. Sex Med Rev 3: 126-136. [Crossref]

5. Virgili A, Corazza M, Minghetti S, Borghi A (2015) Growing Evidence for Topical Mometasone Fuoroate in the Treatment of Vulvar Lichen Sclerosus. Maturitas 80 : 113-115.

6. Murina F, Rehman S, Di Francesco S, Mantegazza V, Felice R, et al. (2015) Vulvar Lichen Sclerosus: A Comparison of the Short-Term Topical Application of Clobetasol Dipropionate $0.05 \%$ versus Mometasone Furoate 0.1. J Low Genit Tract Dis 19: 149151. [Crossref]

7. Fistarol SK, Itin PH (2013) Diagnosis and treatment of lichen sclerosus: an update. $\mathrm{Am}$ J Clin Dermatol 14: 27e47.

8. Chi CC, Kirtschig G, Baldo M, Brackenbury F, Lewis F, et al. (2012) Systematic review and meta-analysis of randomized controlled trials on topical interventions for genital lichen sclerosus. J Am Acad Dermatol 67: 305Y12. [Crossref]

9. Huang X, Tanojo H, Lenn J, Deng CH, Krochmal L (2005) A Novel Foam Vehicle for Delivery of Topical Corticosteroids. J Am Acad Dermatol 53: S26-S38. [Crossref]

Copyright: (C2017 Murina F. This is an open-access article distributed under the terms of the Creative Commons Attribution License, which permits unrestricted use, distribution, and reproduction in any medium, provided the original author and source are credited. 